\title{
Seeking footprints of the primeval Universe in dwarf galaxies
}

\author{
Sebastian L. Hidalgo ${ }^{1,2}$ and the LCID group. \\ ${ }^{1}$ Instituto de Astrofísica de Canarias. Vía Láctea s/n. E38200 - La Laguna, Tenerife, Canary \\ Islands, Spain \\ email:shidalgo@iac.es \\ ${ }^{2}$ Department of Astrophysics, University of La Laguna. Vía Láctea s/n. E38200 - La Laguna, \\ Tenerife, Canary Islands, Spain
}

\begin{abstract}
We present the star formation histories (SFHs) of four isolated dwarf galaxies, Cetus, Tucana, LGS-3, and Phoenix, as a function of galactocentric radius. Our results suggest that beyond some distance from the center, there are no significative differences in fundamental properties of these galaxies, such as the star formation rate (SFR) or age-metallicity relation (AMR). The stellar content of this region would be composed of old ( $\gtrsim 10.5 \mathrm{Gyr}$ ) metal-poor stars only. In the innermost regions, dwarf galaxies appear to have formed stars during time intervals which duration varies from galaxy to galaxy. This extended star formation produces the dichotomy between dwarf spheroidal (dSph) and dwarf Transition (dTr) galaxy types.
\end{abstract}

Keywords. galaxies: dwarf, galaxies: evolution, Local Group.

We present the SFHs of four isolated dwarf galaxies, Cetus, Tucana, LGS-3, and Phoenix, as a function of galactocentric radius. The results are based on the observations of the LCID project (Local Cosmology form the Isolated Dwarfs). The aim of this project is to obtain the full SFHs of six isolated dwarf galaxies of the Local Group: Leo-A, Phoenix, Cetus, Tucana, LGS-3, and IC1613. The global SFHs of these galaxies has been already described in Cole, Skillman, Tolstoy, et al. (2007), Hidalgo, Aparicio, Martínez-Delgado, et al. (2009), Monelli, Hidalgo, Stetson, et al. (2010a), Monelli, Gallart, Hidalgo, et al. (2010b), Hidalgo, Aparicio, Skillman, et al. (2011), and Skillman et al. (2013), respectively. We have selected a subsample of the dwarf galaxies of the LCID project to obtain the $\mathrm{SFH}$ as a function of the galactocentric radius: two dSph galaxies, Cetus and Tucana, and two transition (dTr) galaxies, LGS-3 and Phoenix.

For a clearer and more consistent comparison of the results, four of the regions (R1 to R4) were selected in each galaxy. The three innermost ones are such that their equivalent radii approximately correspond to $\alpha_{\psi}\left(R_{1}\right), 1.5 \alpha_{\psi}\left(R_{2}\right)$, and $2 \alpha_{\psi}\left(R_{3}\right)$ where $\alpha_{\psi}$ is the scale length or the stellar mass distribution of each galaxy. $R_{4}$ corresponds to the area outer of $2 \alpha_{\psi}$. This fourth area has not been used in Phoenix due to the low number of stars observed in it (less than $3 \%$ of the total).

We have used a CMD fitting technique (IAC-star/IAC-pop/MinnIAC) to obtain the SFHs as described in Aparicio \& Hidalgo (2009) and Hidalgo, Aparicio, Skillman, et al. (2011). Figure 1 (left panel) shows the SFR as a function of time, $\psi(t)$, of Cetus, Tucana, LGS-3, and Phoenix obtained for the four regions defined above. The SFR has been normalized to its time integral for each region. There is star formation activity in all gallaxies for ages $\gtrsim 10 \mathrm{Gyr}$, regardless of distance to the center. Interestingly, in the outermost area $\left(R_{4}\right)$ the three normalized SFRs are almost indistinguishable within error bars. However, $\psi(t)$ gradually decreases outwards for ages $\lesssim 9$ Gyr. Few stars younger than this age exist in the $R_{4}$ regions, though some stars can still be observed at $\sim 3.5 \mathrm{Gyr}$ 

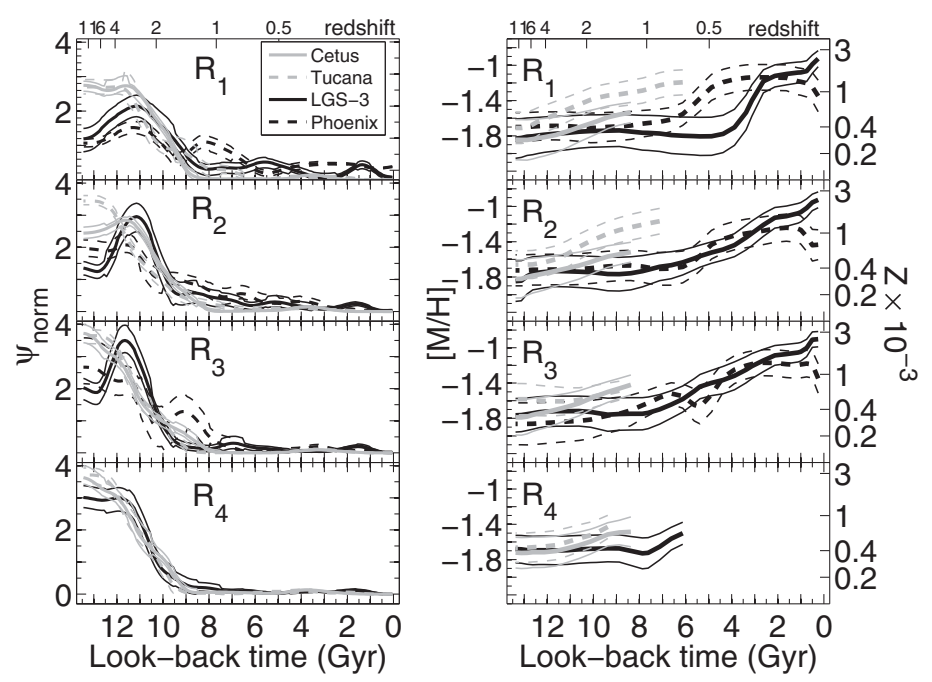

Figure 1. SFHs (left panel) and age-metallicity relations (rigth panel) as a function of galactocentric radius of four isolated dwarf galaxies of the Local Group.

for Cetus and Tucana and at $\sim 1.5$ Gyr for LGS-3. These stars have been identified as a population of Blue-straggler stars (BSS) in Cetus and Tucana by Monelli, Cassis, Mapelli, et al. (2012). In the case of LGS-3, the expected BSSs may be mixed with some young and intermediate age stellar population, which produces the bump at $1.5 \mathrm{Gyr}$ in $\psi(t)$.

Figure 1 (right panel) shows the AMR for the four regions in all the galaxies (three in the case of Phoenix). The overall trend is that metallicity increases with time. However, in the case of LGS-3 and Phoenix, the metallicity remains almost unchanged for most (LGS3) or half (Phoenix) of the lifetime of the galaxy in the central region. For these galaxies, the AMR relation can be separated into two distinct time periods: a first period involving the older stars where metallicity does not change significantly with time, and a final period where the metallicity increases steeply. The duration of the first period seems a function of radius, decreasing with increasing radius. In contrast, the metallicity of Cetus and Tucana increases steadily with time, showing no trend with radius. As a result, stars between $6-10$ Gyr old are on average more metal poor in LGS-3 and Phoenix than in Cetus and Tucana.

\section{References}

Aparicio, A. \& Gallart, C. 2004, AJ, 128, 1465

Aparicio, A. \& Hidalgo, S. L. 2009, AJ, 138, 558

Cole, A. A., Skillman, E. D., Tolstoy, E., et al. 2007, ApJL, 659, L17

Hidalgo, S. L., Aparicio, A., Martínez-Delgado, D., \& Gallart, C. 2009, ApJ, 705, 704

Hidalgo, S. L., Aparicio, A., Skillman, E., et al. 2011, ApJ, 730, 14

Monelli, M., Cassisi, S., Mapelli, M., et al. 2012, ApJ, 744, 157

Monelli, M., Hidalgo, S. L., Stetson, P. B., et al. 2010a, ApJ, 720, 1225

Monelli, M., Gallart, C., Hidalgo, S. L., et al. 2010b, ApJ, 722, 1864

Skillman et al. 2013, in preparation. 